\title{
Intensity Observation of Conjunctivitis using DIP
}

\author{
Jyoti Patil \\ Assistant Professor

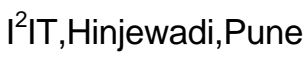

\author{
Anant. L. Chaudhari \\ Head, Department of Electronics \\ MGSM's A.S.C. College, Chopda \\ Dist. Jalgaon 425107 India
}

\begin{abstract}
Medical image processing using MATLAB is used in medical field. The recently many technologies are used for the diagnosis of eye diseases like conjunctivitis to observe infection on eye. This paper Digital image processing (DIP) is applied for manual judgment by doctors to get better \& fast result. The diagnosis of "Intensity of redness of pink Eye" i.e. "Conjunctivitis"; by identifying the region of infection in the corneal images. The eye images are observed in DIP which gives results of the increase in infection or intensity of disease. Considering the importance of digital image processing, our aim is to implement this technology for diagnosis eye diseases. [1]
\end{abstract}

Keywords: DIP, MATLAB, Conjunctivitis, Thresholding

\section{INTRODUCTION}

The conjunctiva is a thin, translucent, relatively elastic tissue layer with both bulbar and palpebral portions. The bulbar portion of the conjunctiva lines the outer aspect of the globe, while the palpebral portion covers the inside of the eyelids. Underneath the conjunctiva lie the episclera, the sclera and the unveil tissue layers (Fig. 1[a]). [1]

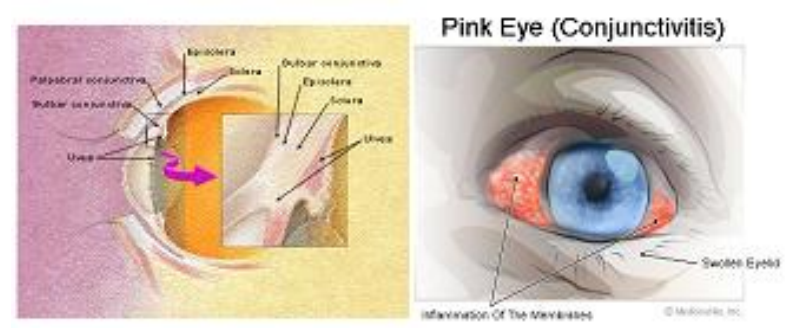

Fig. 1[a]. Anatomy of the eye and eyelids, [b] Conjunctivitis

Conjunctivitis is inflammation of the transparent layer (the conjunctiva) that covers the white of the eye. It is the most common cause of "red eye.", which Causes Vision Loss.

The inflammation can be hyper acute, acute or chronic in presentation and infectious or noninfectious in origin. Symptoms associated with fungal damage, viral diseases, Red eyes were characterized using image-processing techniques. The color analysis showed increase in intensity of infection between the asymptomatic and symptomatic eye. In this paper the infected images of Eye is observe \& also different area of infection of eye is observe. It is find that different areas or points of infection having different intensity level of infection but average or overall intensity of redness of eye is same. Also case of five days of infection is taken, which shows that there is variation in intensity of redness of eye which varies according to diagnosis $\&$ treatment given by doctor. The type of infection decides proper diagnosis. For this threshouldring is best method which detects or counts a number of pixels of colors of image. The whole system is implemented in MATLAB. This article highlights key features in the clinical history and ocular examination that can help family physicians to formulate a differential diagnosis and a management plan for patients with eye diseases. The aim of these researches is to get to know the disease well and to diagnose it more quickly and accurately, with the further goal to work out more effective methods of treatment [2]. Marc M. Schulze et al. in 2000 The Use of Fractal Analysis and Photometry to Estimate the Accuracy of Bulbar Redness Grading Scales, get result of increase in infection or redness in eye.[3]

\section{PRACTICAL WORK: Simple Threshold Method of Segmentation:}

Red eye, clinically known as bulbar hyperemia, is an increased dilation of blood vessels in the bulbar conjunctiva that gives the eye its red appearance and is a prominent sign of ocular irritation. The recognition of change in redness is critical for clinicians in management of the ocular surface, particularly in contact lens research and practice. Hence percentage of redness is estimated with help of following thresholding method, which is helpful to calculate the result to get the pixel values. Thus average value by calculating minimum \& maximum values of number of pixels which is Global thresholding Suppose the intensity of an object is denoted by $f(x, y)$ and the histogram is plot. In this image, it can be seen that the image intensity can be group into two non-overlapping classes based on the value of threshold $\mathrm{T}$ in the histogram. This help in distinguishing the any pixel with value below this threshold, i.e. $\mathrm{f}(\mathrm{x}, \mathrm{y})<\mathrm{T}$ into a class called Background Information and any pixel with intensity value greater than $\mathrm{T}$ i.e. $\mathrm{f}(\mathrm{x}, \mathrm{y})>\mathrm{T}$ into another class called Object. In the segmented binary image, background pixels have value of zero while object pixels have value 1 [4].

$$
g(x, y)= \begin{cases}0 & f(x, y)<T \\ 1 & f(x, y) \geq T\end{cases}
$$

When the value of threshold $\mathrm{T}$ is constant, this is called Global Thresholding. Global thresholding often fails when the background illumination is uneven.

The algorithm works as:

1. Select an initial threshold T0 (e.g. the mean intensity)

2. Partition image into two groups (R1 and R2) using the T0

3. Calculate the mean intensity values $\mu 1$ and $\mu 2$ of the partitions R1 and R2. 
4. Select a new threshold: $\mathrm{Ti}=(\mu 1+\mu 2) / 2$

5 . Repeat steps $2-4$ until: $\mathrm{Ti}=\mathrm{Ti}-1$
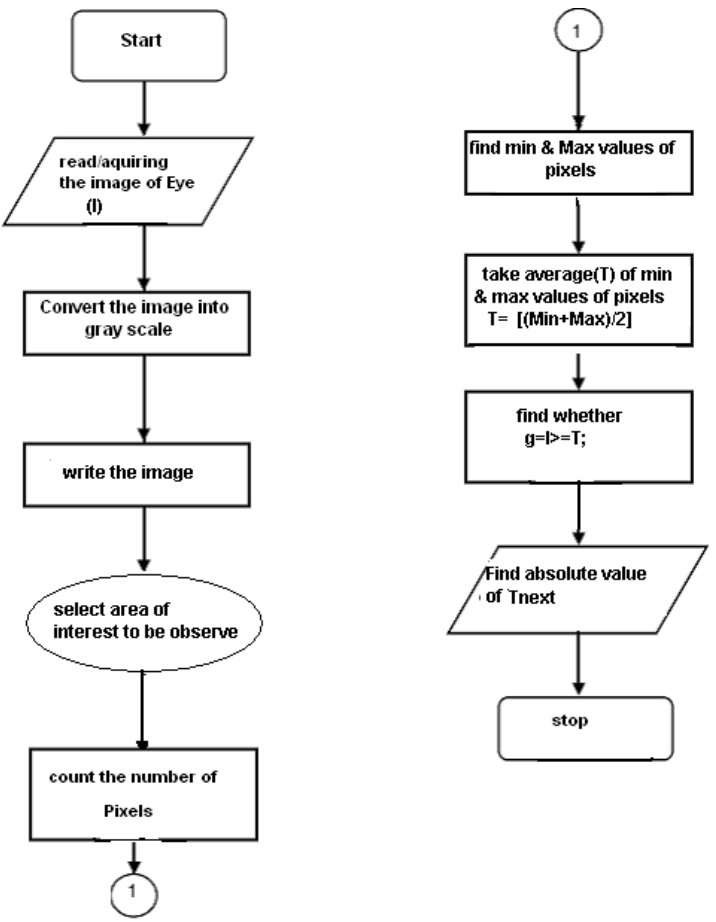

Fig. 2 Flowchart to calculate pixel values

\section{OBSERVATION OF AVERAGE INTENSITY OF HIGHLY INFECTED \\ EYE:}

IRed eye image having 4th or 5th day of high infection is examine to find intensity of redness eye. Any three to four points for observation are chosen to get average intensity of eye. All points give nearly equal value of intensity. To observe intensity Histogram is observed of all three points \& also average value of redness of eye. Firstly three different points of infection of Eye are observed i.e. a points which are are nearest integer value of redness $(\mathrm{x}, \mathrm{y})$, which gives number of infected pixels. [5]

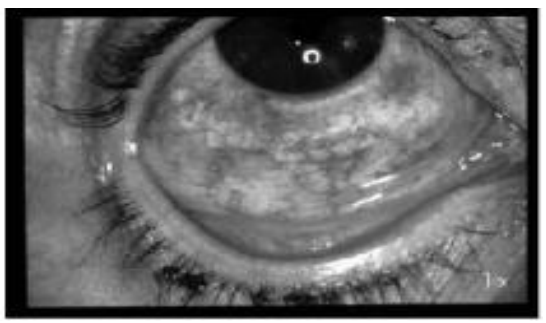

Fig[3] Infected image of eye

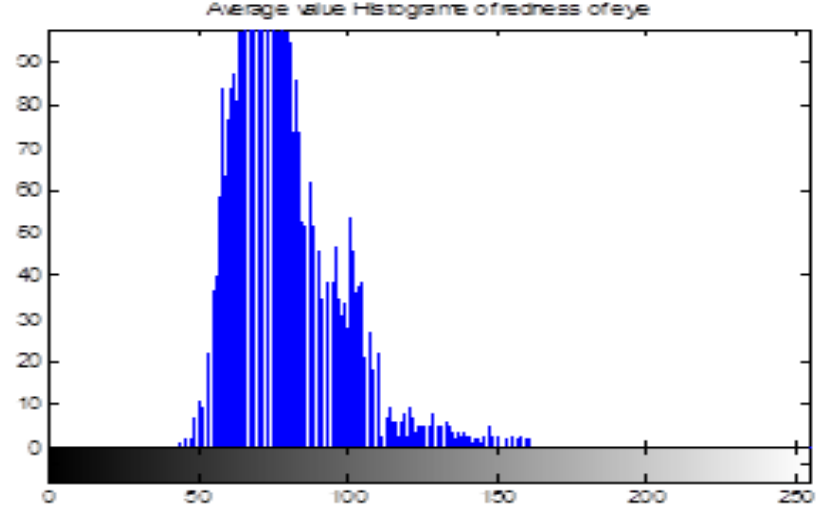

Fig.[4 ] Histogram of Average value of redness of eye

Average Intensity of redness is nearly $=49.2$

The three different points are selected which are highly intensive with conjunctivitis.

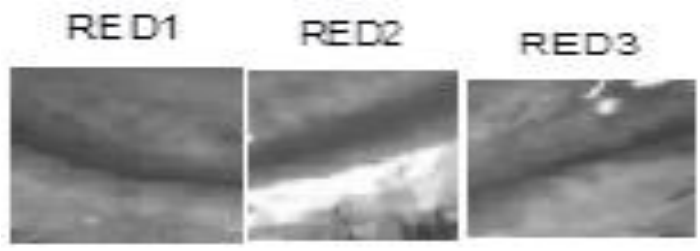

Fig.[5] Three different points

Observation of intensity of redness of three points: selected three points of eye whose pixels values need to find Intensity of redness of eye.

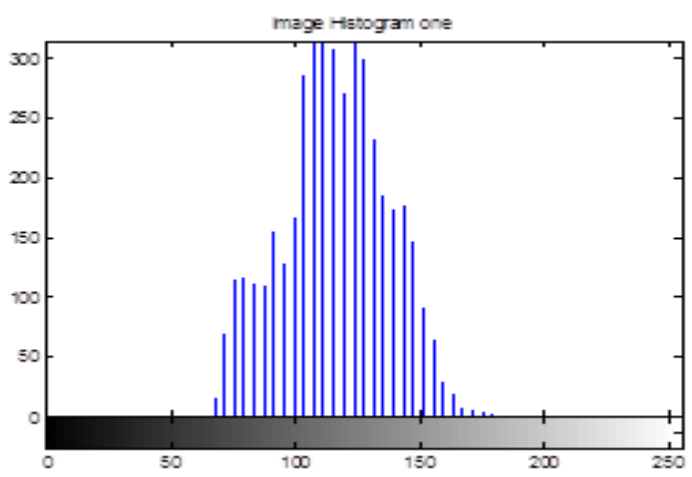

Fig.[6] Histogram of showing value of redness of eye of point

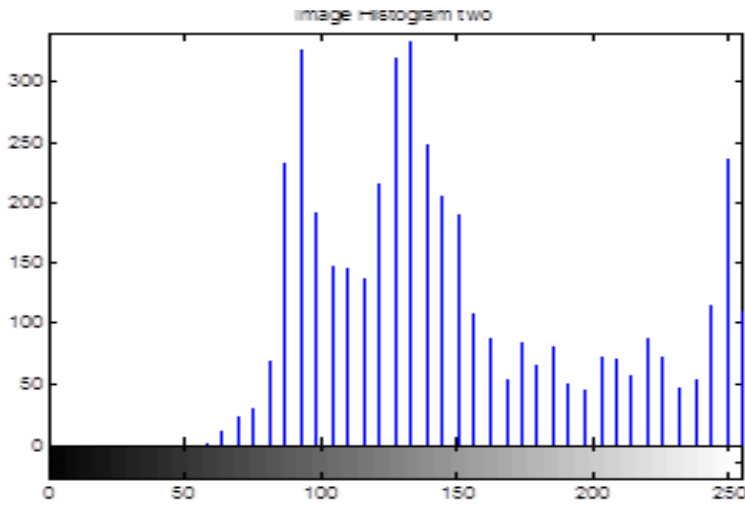

Fig.[7] Histogram showing value of redness of point II $^{\text {nd }}$ 


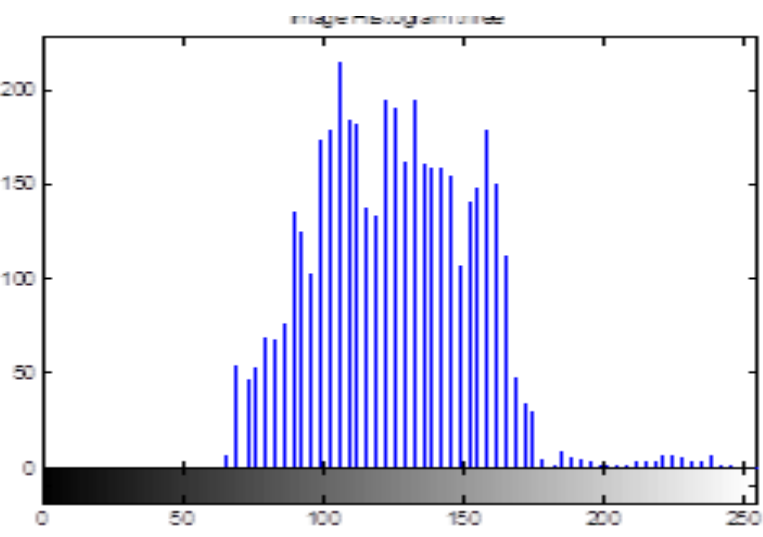

Fig. [8] Histogram showing value of redness of point III ${ }^{\mathrm{re}}$

The above Fig.(6),(7),(8) shows the Histogram of area of infection. The histogram result shows the intensity percentage of redness of eye at three different points of eye

point $\mathrm{I}=46.7 \%$,point $\mathrm{II}=47.8 \%$,point $\mathrm{III}=49.9 \%$

Thus this shows that average value of Intensity of overall area is equal to the calculated pixel values of intensity of three different points.

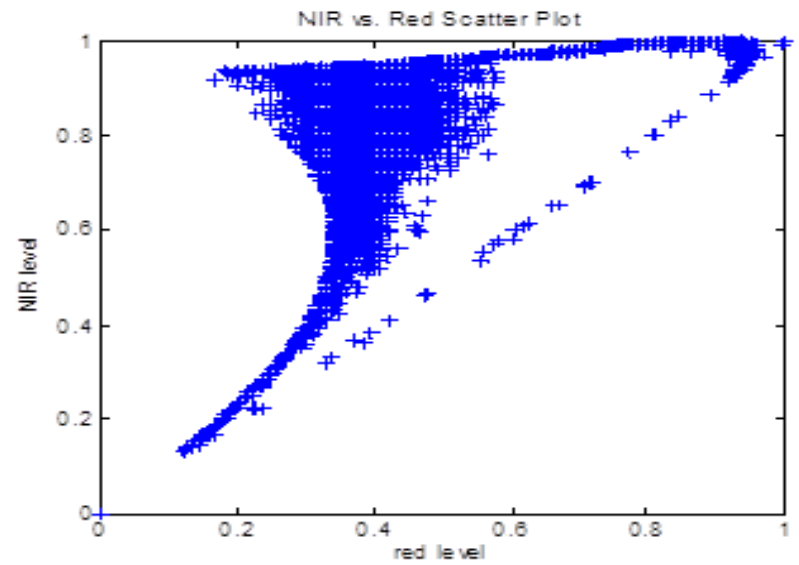

Fig[9] Scatter plot of red color

\section{OBSERVATION OF GROWTH IN CONJUNCTIVITIS WITH INCREASE IN DAY OF INFECTION:}

In our work thresholding, enhancement, Segmentation and registration techniques are used. Observe photograph of infected Eye for number of days of infection, result Shows that intensity of infection increases as number of days passes. These results help to get Intensity values of redness of eye for which the threshouldring is the best method which detects or counts a number of pixels of colors of image.
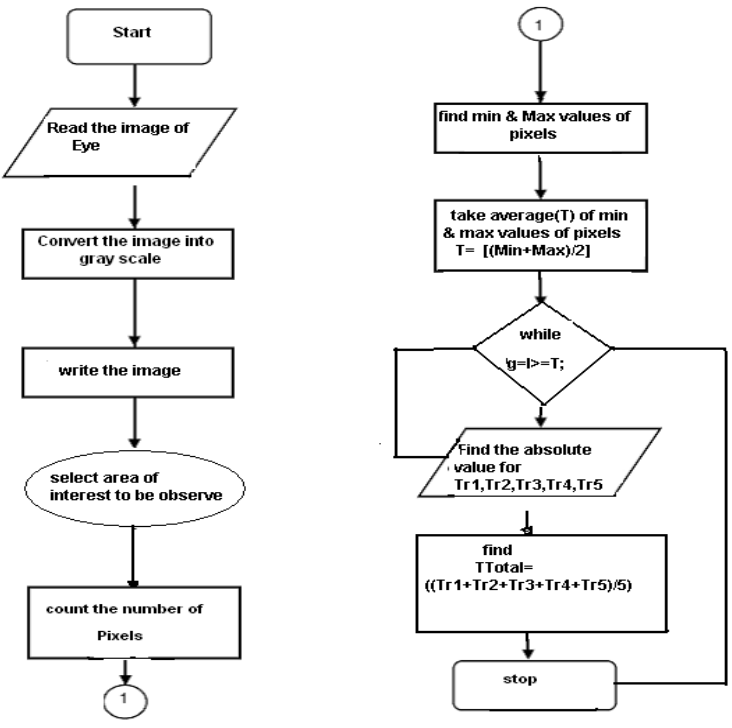

Fig.[10] Flowchart to calculate absolute value

The aim of paper is to get the value of intensity of redness of eye disease Conjunctivitis. These report observe the infection of disease, thus five images for five days of infection has been observed.

\section{First day of Infection of Conjunctivitis:}

Fig. [11] shows first day infection shows the initial stage of infection where it observe that for first day infection is minor. And the value of intensity of redness is $\operatorname{Tr} 1=0.4392$.
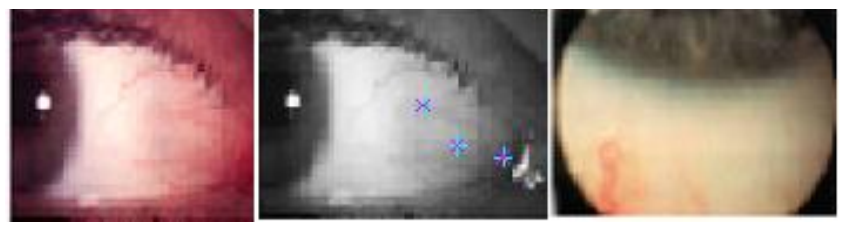

Fig,[11] first day of infection

Intensity value of redness of eye first day $\operatorname{Tr} 1=$ 0.4392

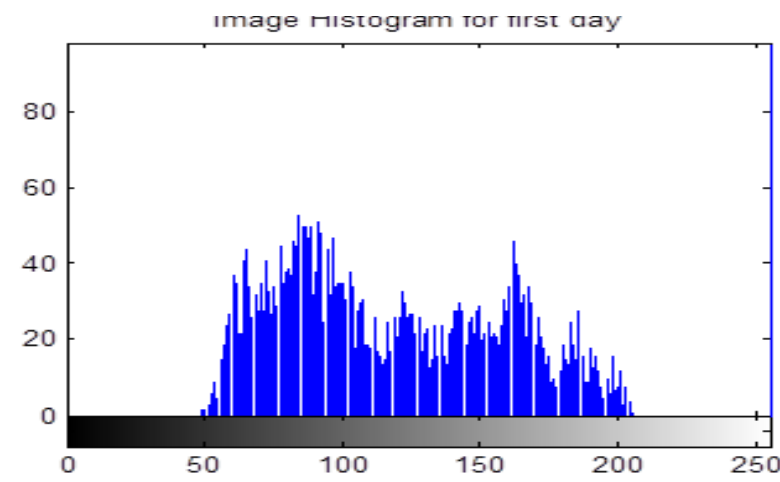

Fig.[11-a] Histograme for the first day of infection.

Second day of Infection of Conjunctivitis: Fig.[12] shows the image of infection of second day it represents the value of redness $\operatorname{Tr} 2=0.5098$ which is more than first day of infection. 


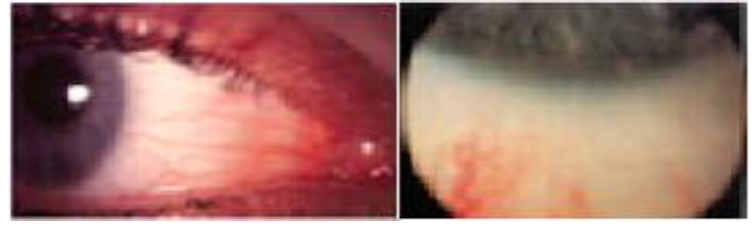

Fig. [12] second day of infection

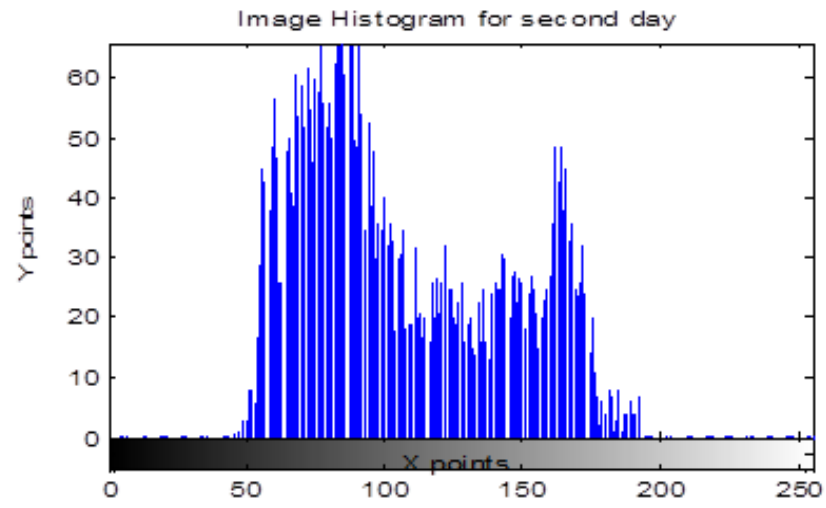

Fig.[12-a] Histogram of second day of infection.

Intensity value of redness of eye second day $\operatorname{Tr} 2=$ 0.5098

\section{Third day of Infection of Conjunctivitis:}

Fig.[13] shows Third day of infection of third day it represents the value of redness $\operatorname{Tr} 3=0.6471$ which more than second day of infection.

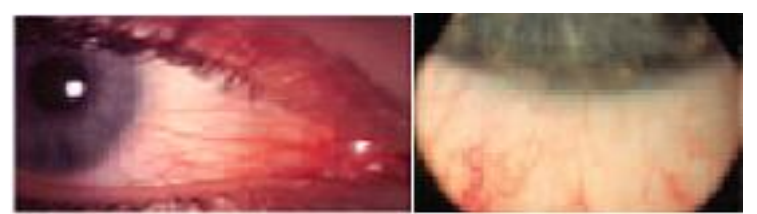

Fig.[13] Third day of infection

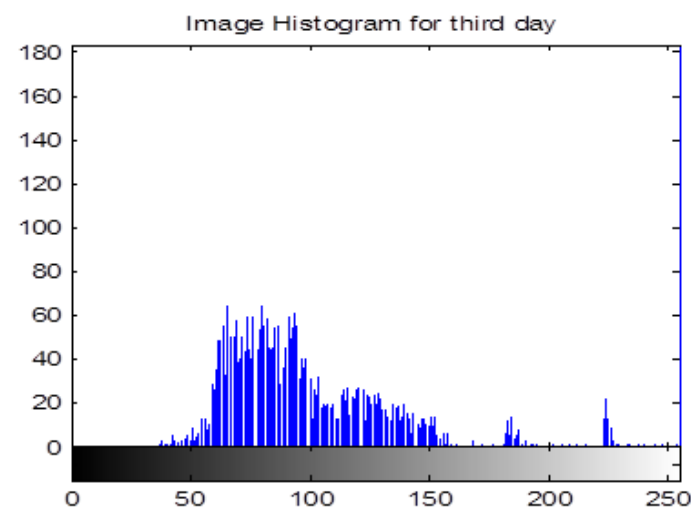

Fig.[13-a] Histogram of second day of infection

Intensity value of redness of eye third day $\operatorname{Tr} 3=$ 0.6471

\section{Fourth day of Infection of Conjunctivitis:}

Fig.[14] shows fourth day it represents the value of redness $\operatorname{Tr} 4=0.6686$ which more than third day of on intensity of infection, which Maximum Intensity of Infection.

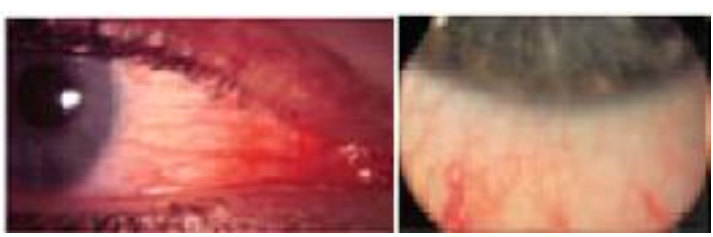

Fig.[14] fourth day of infection

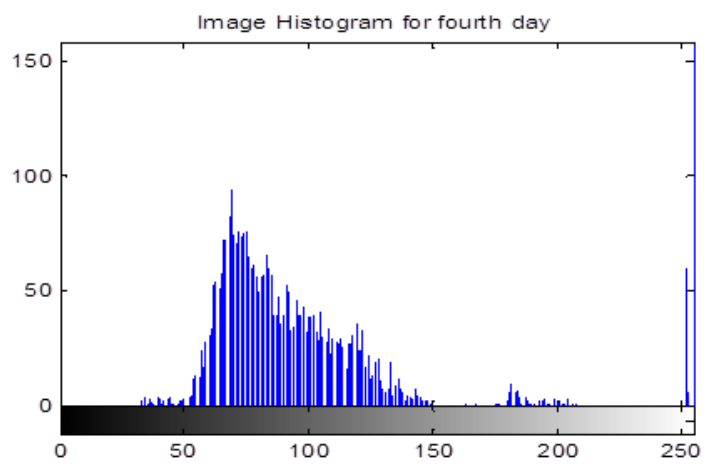

Fig.[14-a] Histogram of second day of infection

Intensity value of redness of eye fourth day $\operatorname{Tr} 4=$ 0.6686.

Fifth day of Infection of Conjunctivitis:

Fig.[15] is fifth day of infection which same as fourth day having value of redness $\operatorname{Tr} 5=0.6686$ which Maximum Intensity of Infection.

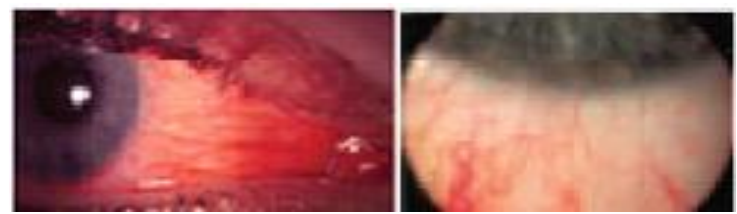

Fig.[15] fifth day of infection

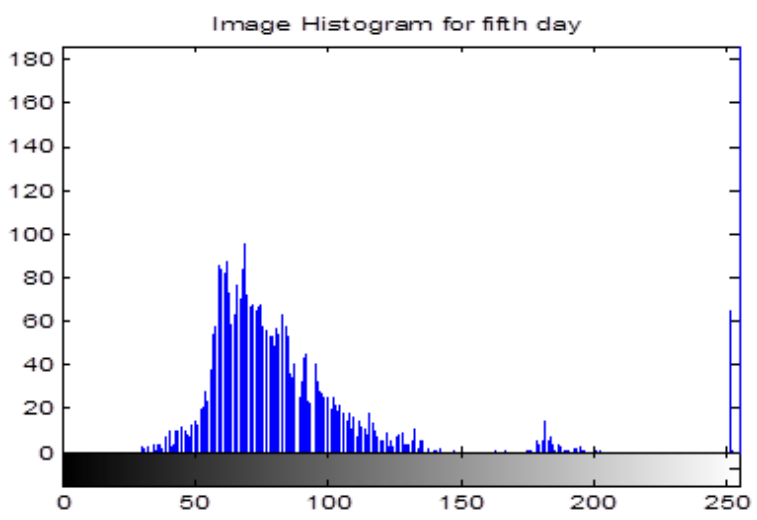

Fig. [15-a] Histogram of second day of infection 
Intensity value of redness of eye fifth day $\operatorname{Tr} 5=$ 0.6686. Thus redness and Intensity of eye increases with increase in days of infection.

\section{Average Days of Intensity $=\mathbf{T}$ Total $=\mathbf{0 . 5 8 6 6 6 6}$}

Thus from above observation it is conclude that intensity of redness of eye increases With increase in days. The largest conjunctival area possible of each reference image, the results for the individual segmented Region Of Interest is measure.averaged to represent a global estimate of the conjunctival redness.

\section{RESULT}

Thus to calculate the redness of eye the number of red pixels are count. Thus suppose for the segmentation of image is best method in image processing using which the number of pixels can be easily count. The first goal of this study was to find the intensity or the to count number red pixels of infected Of red/pink eye, which is done using Globle thresholding method.

a) Observe average intensity of higly infected eye which compare with three infected points of the same eye which observe to be same. which is nearly $\mathbf{4 9 . 2}$

b) Observe Increase in Intensity of redness of eye with increase in days of infection in which we observe that from first day to fifth day intensity of redness increases. So with help of we can observe percentage of redness. which is not possible to observe manually so using DIP we have detect the retinal image, \& after application of thresholding ophthalmologist can easily observe the damage nerve fibers [6].
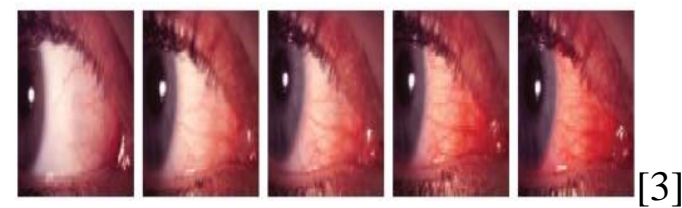

1)Day 1st $=0.4392$, 2) Day 2nd $=0.5098,3$ ) Day 3rd $=0.6471,4$ ) Day 4th $=0.6686,5$ ) Day 5 th $=0.6686$.

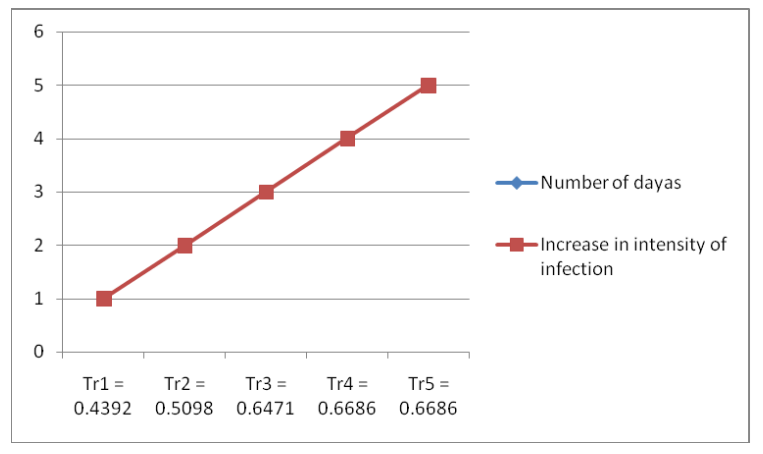

Fig.[j] increase in intensity of redness with number of days.
Average Days of Intensity $=\mathbf{T}$ Total $=\mathbf{0 . 5 8 6 6 6 6}$

\section{REFERENCES:}

[1] GARY L. MORROW, M.D., Toronto East General and Orthopedic Hospital, Toronto, Ontario, Canada RICHARD L. ABBOTT, M.D., University of California, San Francisco, and Francis I. Proctor Foundation, San Francisco, California.

[2] Madhusudhanan Balasubramanian B.E., Bharathiyar University, 1998, " COMPUTER ASSISTED EYE FUNGAL INFECTION DIAGNOSIS".

[3] The Use of Fractal Analysis and Photometry to Estimate the Accuracy of Bulbar Redness Grading Scales, by Marc M. Schulze et al.

[4] Reference from http://en.wikipedia.org/wiki/ Edge_detection

[5] LIXU GU, SEMINAR SERIES ON ADVANCED MEDICAL IMAGE PROCESSING(2), FUNDAMENTAL CONCEPTS Robarts Research Institute London, Ontario, Canada, July 10, 2002.

[6] Rafael C. Gonzalez and Richard E. Woods. 'Digital Image Processing using MATLAB',2nd edition. Prentice Hall, 2002. ISBN 0-201-18075-8.

[7] Marc M. Schulze, Natalie Hutchings, and Trefford L. Simpson1, The Use of Fractal Analysis and Photometry to Estimate the Accuracy of Bulbar Redness Grading Scales”, IOVS, April 2008, Vol. 49, No. 4

\section{AUTHOR'S PROFILE}

Ms.Jyoti Devidas Patil. Born in 1983, obtained M. Sc., M. Phil. Degree from North Maharashtra University, Jalgaon. Joined the Department of Physical Science. PresentlyWorking as Assistant Professor in Engineering Science Department of "International Institute of Information Technology, Pune".Currently working on Image Processing.

Dr. A. L. Chaudhari, born in 1964, obtained M. Sc., M. Phil. $\& \mathrm{Ph} . \mathrm{D}$. degrees from University of Pune. Joined the Department of Electronics, Arts, Science \& Commence college, Chopda Dist. Jalgaon, India as lecturer since 1988 \& presently working as Associate Professor, Head of Electronics $\&$ Computer science department. He is guiding students for M. Phil. \& Ph.D .Co-authored 10 books \& published 25 papers in national \& international journals/conference proceedings. Visited National university of Singapore \& University of Tokushima, Japan. He is executive council member of IETE(Pune Chapter),member of Board of Studies \& member of Faculty of Science, North Maharashtra University, Jalgaon Completed minor research project sponsored by UGC. His current area of research is fiber optic sensor, PC based instrumentation \& image processing. 